\title{
Probing Interaction of Charged Nanoparticles with Uncharged Micelles
}

\author{
Sugam Kumar ${ }^{1}$, V.K. Aswal ${ }^{1, *}$ and J. Kohlbrecher ${ }^{2}$ \\ ${ }^{1}$ Solid State Physics Division, Bhabha Atomic Research Centre, Mumbai 400 085, India \\ ${ }^{2}$ Laboratory for Neutron Scattering, Paul Scherrer Institut, CH-5232 PSI Villigen, Switzerland \\ *E-mail:vkaswal@barc.gov.in
}

\begin{abstract}
Small-angle neutron scattering (SANS) and dynamic light scattering (DLS) studies have been carried out to study interaction of anionic nanoparticles with uncharged (non-ionic) C12E10 micelles. DLS results show increase in the effective size of nanoparticles on addition of C12E10. Contrast variation SANS confirms the micelles decoration of nanoparticles which is believed to be driven by the hydrogen bonding between them. The number of adsorbed micelles per nanoparticle increases with the increase in the nanoparticle size. Surface number density is also found to be increasing with increase in particle size indicating favored adsorption for larger curvature.
\end{abstract}

Keywords: Self-assembly, Nanoparticle-surfactant interaction, SANS

PACS: 61.05.fg, 64.70pv, 64.75.Yz

\section{INTRODUCTION}

Nanoparticles and their complexes with macromolecules are being utilized in the areas of nanobiotechnology and nanoengineering [1]. The interaction and resultant structure is decided by the properties of nanoparticles as well that of macromolecule. Different macromolecules that can be used are protein, polymers and surfactant, etc. Applications such as drug delivery, cosmetics, enhanced dispersion stability, involve interfacing of nanoparticles with surfactant. The driving force in these systems is cumulative effect of covalent bonding, electrostatic forces, hydrogen bonding etc [2]. In case of nonionic surfactants, where the electrostatic attraction is absent, interaction occurs mainly due to hydrogen bonding of ether oxygen of the ethylene oxide group and the surface $\mathrm{OH}$ group. Such interaction may lead to the bilayer formation or direct adsorption of micelles on the nanoparticles as known in the literature [3]. The large surface-to-volume ratio, curvature and availability of functional groups on the surface are important parameters for composite formation governed by nanoparticle size. In this work, we have studied the interaction of uncharged micelles with different sized anionic silica nanoparticle using DLS and SANS techniques. In particular, SANS with the possibility to vary contrast of individual components, is an ideal technique to study such multi-component systems.

\section{EXPERIMENTAL}

Electrostatically stabilized colloidal suspensions of three sizes of silica nanoparticles (Ludox SM30, HS40 and TM40) and non-ionic surfactant C12E10 (decaoxyethylene $n$-dodecylether) were obtained from Sigma-Aldrich. SANS experiments were carried out at SANS-I facility, Swiss Spallation Neutron Source SINQ, Paul Scherrer Institut, Switzerland [4]. Data were collected at two sample-to-detector distances 2 and $8 \mathrm{~m}$ to cover a $Q$ range of 0.008 to $0.3 \AA^{-1}$. Corrections were made for background and empty cell contributions and data were normalized to absolute cross-sectional unit using standard procedure. Dynamic light scattering (DLS) experiments were performed using an Autosizer 4800 (Malvern Instruments, UK) operating with digital correlator and Ar-ion laser source operated at wavelength $514.5 \mathrm{~nm}$.

\section{SANS ANALYSIS}

In SANS experiments, one measures the coherent differential scattering cross section per unit volume $(\mathrm{d} \Sigma / \mathrm{d} \Omega)$ as a function of $\mathrm{Q}$ and is given by

$$
\frac{d \Sigma}{d \Omega}(Q)=n V^{2}\left(\rho_{p}-\rho_{s}\right)^{2} P(Q) S(Q)+B
$$

where $n$ is number density of particles and $V$ is particle volume. $\rho_{p}$ and $\rho_{s}$ are scattering length densities of 
particle and solvent, respectively. $P(Q)$ is intraparticle structure factor and depends on shape and size of the particle. $S(Q)$ is interparticle structure factor and is unity for diluted system. $B$ is a constant term representing incoherent background.

\section{RESULTS AND DISCUSSION}

Fig. 1 shows the SANS data of $1 \mathrm{wt} \%$ from three sized silica nanoparticles SM30 (10 nm), HS40 $(16 \mathrm{~nm})$ and TM40 (26 nm) with $1 \mathrm{wt} \% \mathrm{C} 12 \mathrm{E} 10$ surfactant. Data for all three nanoparticle systems show similar features at higher $\mathrm{Q}$ region as the scattering in this region is decided by surfactant micelles (free or adsorbed). On the other hand, it can be seen that there is size dependent systematic increase of scattering in the low Q region where the scattering is governed by size of the nanoparticle. The build-up of scattering is found significantly more compare to the addition of the two pure components (inset I of the Fig. 1) indicating the formation of larger structures. This has also been confirmed by DLS measurements where calculated hydrodynamic size of nanoparticles increases from 17.0 to $30.6 \mathrm{~nm}$ on interacting with surfactant. The measured auto correlation functions of $1 \mathrm{wt} \%$ HS40 nanoparticles without and with $1 \mathrm{wt} \%$ $\mathrm{C}_{12} \mathrm{E}_{10}$ micelles are shown in inset II of Fig.1. The formation of larger structures can be explained by two models known in literature: bilayer formation on the nanoparticle surface or decoration of nanoparticles by micelles directly [5].

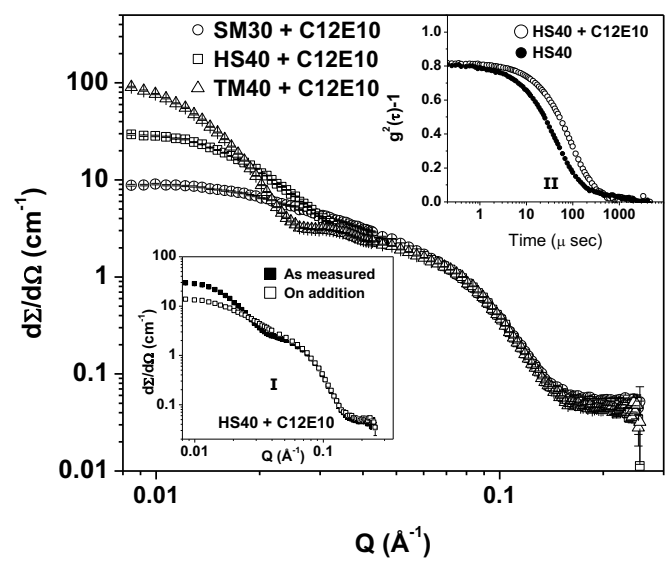

FIGURE 1. SANS data of $1 \mathrm{wt} \%$ silica nanoparticles with $1 \mathrm{wt} \% \mathrm{C}_{12} \mathrm{E}_{10}$ surfactant. Inset I shows comparison of data for mixed system to the addition of pure components. Auto correlation functions of $1 \mathrm{wt} \%$ HS40 without and with $1 \mathrm{wt} \% \mathrm{C}_{12} \mathrm{E}_{10}$ are shown in inset II.

Contrast variation SANS data support to the model of micelles adsorbed on the nanoparticle surface [6]. To simplify these systems, silica nanoparticles are contrast-matched to the solvent (Fig. 2), where the scattering is governed by adsorbed micelles and free micelles (if any). It is found that the number of adsorbed micelles per nanoparticle increases drastically whereas the percentage of adsorbed micelles on nanoparticles decreases with the increase in the size of nanoparticles. The increase in number of adsorbed micelles per nanoparticles is 3 to 104 and decrease in fraction of adsorbed micelles is 70 to $23 \%$ when the size of nanoparticle increases from 10 to $26 \mathrm{~nm}$. The fraction of adsorbed micelles depends on the surface available to micelle adsorption, which decreases with the increase in the nanoparticle size at fixed concentration of the particles. Surface number density is found to increase 0.014 to $0.049 \mathrm{~nm}^{-2}$ with nanoparticles size increasing 10 to $26 \mathrm{~nm}$ indicating favored adsorption for larger curvature. Further, any interactional changes in the nanoparticles due to the presence of these micelles are examined by contrast matching surfactant to the solvent. SANS data of nanoparticle-C12E10 system (micelles are contrastmatched) are found to be identical to that of pure nanoparticle systems in same contrast condition (inset of Fig.2) suggesting absence of any interactional or structural changes in the nanoparticles.

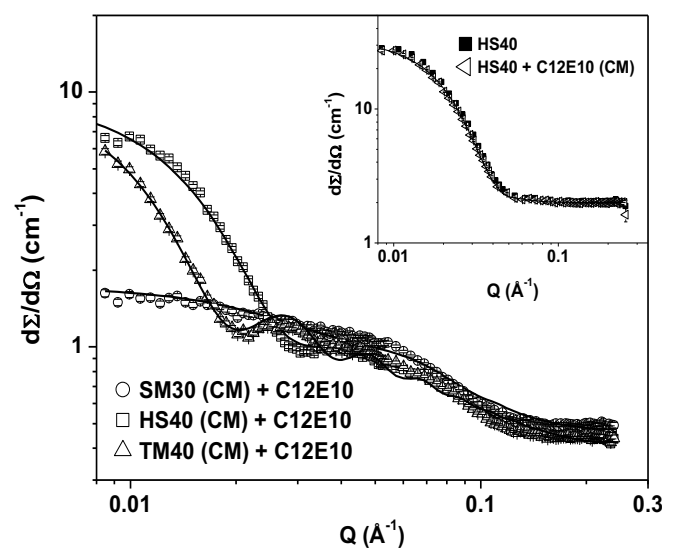

FIGURE 2. SANS data of $1 \mathrm{wt} \%$ silica nanoparticles with $1 \mathrm{wt} \% \mathrm{C}_{12} \mathrm{E}_{10}$ surfactant when silica is contrast-matched. Inset shows SANS data of HS40 nanoparticles without and with surfcatant when surfactant is contrast-matched.

\section{REFERENCES}

1. W. H. Suh, Y.H. Suh and G.D. Stucky, Nano Today, 4, 27-36 (2009)

2. R. Zhang and P. Somasundaran, Adv. Colloid Interface Sci. 123-126, 213-229 (2006).

3. Sugam Kumar, V.K. Aswal and J. Kohlbrecher Langmuir 28, 9288 - 9297 (2012)

4. J. Kohlbrecher and W. Wagner, J. Appl. Cryst. 33, 804-806 (2000)

5. D. Lugo, J. Oberdisse, M. Karg, R. Schweins and G. H. Findenegg, Soft Matter 9, 2928-2936 (2009).

6. Sugam Kumar and V.K. Aswal, J. Phys.: Condensed Matt. 23, 035101 (2011) 